\title{
An improved Gabor filtering for fingerprint image enhancement technology
}

\author{
Hongchang $\mathrm{Ke}^{1, \mathrm{a}}$, Hui Wang ${ }^{2, \mathrm{~b}}$ and Degang Kong ${ }^{1, \mathrm{c}}$ \\ ${ }^{1}$ Software Vocational Institute of Technology, Changchun Institute of Technology, \\ Changchun 130012, China \\ ${ }^{2}$ College of Computer Science and Engineering, Changchun University of Technology, \\ Changchun 130012, China \\ akehongchang1981@163.com, 'banghui1982@mail.ccut.edu.cn, 'ckongdegang2011@sohu.com
}

Keywords: fingerprint identification, image enhancement, band-pass filtering, low-pass filtering

\begin{abstract}
The effect of image enhancement has an impact on features extraction and the fingerprint identification of recognition rate, according to the research of traditional fingerprint image enhancement technology, an improved Gabor filtering for fingerprint image enhancement technology is proposed, using orientation selection and frequency selection characteristics of Gabor filtering, local orientation of fingerprint image and ridge line frequency are the parameter of Gabor filtering function, the two-dimensional Gabor filter is divided into a one-dimensional band-pass filtering and a one-dimensional low-pass filtering. The algorithm has increased computing speed and efficiency and has a good robustness.
\end{abstract}

\section{Introduction}

In the early 1990's, as development of social information fingerprint identification began to apply in civilian area, such as fingerprint entrance guard, fingerprint attendance, fingerprint encryption, fingerprint in bank, fingerprint in social security and fingerprint in id card and so on[1]. The application of the fingerprint identification puts forward to higher requirement, mainly reflected in a higher degree of automation, higher identification accuracy and identification speed. The higher requirement brings more challenge to the research of fingerprint identification technology[2].

In practice, affected by various factors the collected fingerprint images can not guarantee that all are clear, the image may appear in ridge adhesions, ridge fracture or contrast uneven and so on. In this case it is difficult to properly separate from the fingerprint ridge, fingerprint feature extraction is become very difficult. Therefore, before the fingerprint feature extraction and matching the fingerprint image enhancement processing are necessary. Fingerprint image enhancement is using the certain algorithm to make the texture clear, try to highlight and retain the inherent fingerprint feature information, eliminate noise and avoid false features. At present there are lots of research on fingerprint image enhancement algorithm[3-4]. In the present fingerprint image enhancement algorithms, Hong and others proposed based on the Gabor filtering method can obtain relatively good results, which is considered to be the most popular method[5]. This is mainly because the Gabor filtering having optimal joint space-frequency resolution and having a better combination of fingerprint features orientation and frequency information.

Based on the traditional fingerprint image enhancement research, an improved Gabor filter for fingerprint image enhancement technology is proposed in the paper, using Gabor filtering orientation selection and frequency selection characteristic, the orientation and the local ridge frequency of fingerprint image are parameters of the Gabor filter function, the two-dimensional Gabor filtering is divided into a one-dimensional band-pass filtering and a one-dimensional low-pass filtering.

\section{Fingerprint image enhancement method}


Fingerprint image enhancement includes determination of the fingerprint orientation field, determination of the ridge frequency and filtering enhancement

Determination of the fingerprint orientation field. Orientation field reflects the orientation of fingerprint image ridges, its accuracy directly influences the effect of image enhancement[7]. According to the characteristics of ridge direction in local area basically consistent, the fingerprint image is divided into some blocks, then calculating the ridge orientation of each block, finally the ridge orientation represents the orientation of the each pixel in each block. With this method, the fingerprint orientation field has a high efficiency and less amount of image noise. The algorithm is as follows:

Step1: firstly, the image is divided into $w \times w$ non-overlapped blocks,

Step2: using the sobel gradient operator to calculate gradient vector of each pixel in fingerprint image $[G x, G y]^{T}, G x$ represents the horizontal gradient component, Gy represents the vertical gradient component. Then expand the direction of the gradient component one time, $\left[G x^{\prime}, G y^{\prime}\right]^{T}$ vector can be obtained, then

$$
\left[\begin{array}{l}
G x^{\prime} \\
G y^{\prime}
\end{array}\right]=\left[\begin{array}{l}
G x^{2}-G y^{2} \\
2 G x G y
\end{array}\right]
$$

Step 3: Find the average gradient vector $\left[\overline{G x^{\prime}}, \overline{G y^{\prime}}\right]^{T}$,

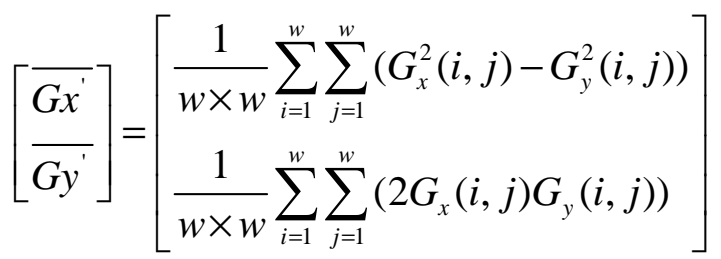

Where $w \times w$ is the size of the local neighborhood.

Step 4: According to the direction of average gradient, find $\varphi$, which is the ridge direction of each pixel.

$$
\varphi=\left\{\begin{array}{lc}
\frac{1}{2} \tan ^{-1} \frac{\overline{G_{y}^{\prime}}}{\overline{G_{x}^{\prime}}}+\frac{\pi}{2} & \tan ^{-1} \frac{\overline{G_{y}^{\prime}}}{\overline{G_{x}^{\prime}}}<0 \\
\frac{1}{2} \tan ^{-1} \frac{\overline{G_{y}^{\prime}}}{\overline{G_{x}^{\prime}}}-\frac{\pi}{2} & \tan ^{-1} \frac{\overline{G_{y}^{\prime}}}{\overline{G_{x}^{\prime}}} \geq 0
\end{array}\right\}
$$

Determination of the ridge frequency. Fingerprint ridge frequency decides the filtering effect. If frequency selection is not appropriate, it will make the filtering images to produce a great deformation, result in the structure of some ridges are suppressed, so that after filtering the fingerprint image has the vacancy.

According to the ridge orientation, determine the orientation window, project each pixel in the window at the projective baseline, analysis projection results the distance between the peak and the trough, then the ridge frequency information can be obtained. The algorithm is as follows:

Step1: firstly, The image is divided into $w \times w$ non-overlapped blocks,

Step2: For each block centered at pixel $(x, y)$, compute an $l \times w$ oriented window, where $l$ along the gradient direction, and $l=32, w$ along the ridge direction of the fingerprint point $(i, j)$, and $w=16, w$ is greater than the width of the block

Step3: calculate the mean value of each point along the $w$ direction, denoted by $M[k]$;

Step4: if the $l \times w$ window without singular point, $M[k]$ forms a discrete sin wave, the frequency is the same as the fingerprint ridge frequency. Let $V(i, j)$ is the number of pixels in $M[k]$ between two continuous peak, then the fingerprint ridge frequency is $f(i, j)=1 / V(i, j)$, if there is no peak, frequency is 0 . 
Filtering enhancement. Gabor filtering is one of the most common fingerprint enhancement algorithm. The principle of the algorithm is based on the mathematical model of fingerprint, in the local area fingerprint can be thought of as a set of parallel with certain frequency linear, along the ridge orientation using the Gabor window function to filter the image, so that the ridge information was enhanced. Because it is along the ridge orientation to filter, which along the ridge line has a smooth function, so that some fault ridge can be repaired to its original state; at the same time the Gabor filtering has good frequency selectivity, it can effectively remove the noise on the ridge and keep the ridge structure.

The mathematical expression of Gabor function is shown as eq. 4:

$$
G(x, y)=\frac{1}{2 \pi \sigma_{x} \sigma_{y}} \exp \left[-\frac{1}{2}\left(\frac{x^{2}}{\sigma_{x}^{2}}+\frac{y^{2}}{\sigma_{y}^{2}}\right)\right] \exp (-2 \pi f i x)
$$

By eq. 4 can be seen, in the $x$ direction, Gabor filter is a band-pass filtering, $y$ direction is a low-pass filtering. When filtering, filter only need rotating, in according with the fingerprint orientation, which can make the fingerprint information greatly enhancing, vertical to the ridge orientation information is weakened relatively.

According to Euler's formula, get the real part $R(x, y)$ and imaginary part $V(x, y)$ of $G(x, y)$

$$
\begin{aligned}
& R(x, y)=\frac{1}{2 \pi \sigma_{x} \sigma_{y}} \exp \left[-\frac{1}{2}\left(\frac{x^{2}}{\sigma_{x}^{2}}+\frac{y^{2}}{\sigma_{y}^{2}}\right)\right] \cos (2 \pi f x) \\
& V(x, y)=\frac{1}{2 \pi \sigma_{x} \sigma_{y}} \exp \left[-\frac{1}{2}\left(\frac{x^{2}}{\sigma_{x}^{2}}+\frac{y^{2}}{\sigma_{y}^{2}}\right)\right] \sin (2 \pi f x)
\end{aligned}
$$

It is obviously to see that $R(x, y)$ is an even function, in the region the current point $(x, y)$ center symmetry, the information of $(x, y)$ will be enhanced,

Therefore, the general form of even symmetric Gabor filtering is as follows:

$$
\begin{aligned}
& R(x, y, f, \varphi)=\frac{1}{2 \pi \sigma_{x} \sigma_{y}} \exp \left[-\frac{1}{2}\left(\frac{x_{\varphi}{ }^{2}}{\sigma_{x}^{2}}+\frac{y_{\varphi}{ }^{2}}{\sigma_{y}^{2}}\right)\right] \cos \left(2 \pi f x_{\varphi}\right) \\
& \text { In eq. 7, }\left[\begin{array}{c}
x_{\varphi} \\
y_{\varphi}
\end{array}\right]=\left[\begin{array}{cc}
\cos \varphi & \sin \varphi \\
-\sin \varphi & \cos \varphi
\end{array}\right]\left[\begin{array}{l}
x \\
y
\end{array}\right] \text { where } \varphi \text { is the orientation of the Gabor filter, } f \text { is the }
\end{aligned}
$$

frequency of a sinusoidal plane wave, $\left[x_{\varphi}, y_{\varphi}\right]$ stands for axis $[x, y]$ along the counterclockwise rotation $\varphi$ degrees:

\section{An improved Gabor filtering}

The Gabor filtering is applied to the fingerprint image enhancement, which need to define four parameters in eq. 7, According to section 2, the ridge orientation and ridge frequency can be calculated, $f, \varphi$ can be obtained, $f$ is the fingerprint ridge frequency, $\varphi$ is the fingerprint orientation, after the test, the value of $\sigma_{x}$ and $\sigma_{y}$ is 6 ,

$$
E(x, y)=\sum_{u=-w}^{w} \sum_{v=-w}^{w} h(u, v: O(x, y), F(x, y)) G(x-u, y-v)
$$

Where $E(x, y)$ is filtering image, $O(x, y)$ is the orientation image, $F(x, y)$ is the frequency image, $G(x, y)$ is the normalization image. The value of $w$ is 5 .

Through eq(8) can be seen, the time complexity of the algorithm is high, in order to improve the efficiency of the algorithm, an improved Gabor filtering algorithm is proposed in this paper, two-dimensional Gabor filtering is divided into a one-dimensional band-pass filtering and a one-dimensional low-pass filtering. The algorithm is as follows:

Step1: the fingerprint image is divided into $w \times w$ non-overlapping blocks, 
Step2: with each block for the center to expand, the size of expansion block is $(w+l) \times(w+l)$, where $l$ is the length of one-dimensional filtering;

Step3: the direction of the block $\varphi$ are $0^{\circ}, 22.5^{\circ}, 45^{\circ}, 67.5^{\circ}, 90^{\circ}, 102.5^{\circ}, 135^{\circ}, 157.5^{\circ}$,

Step4: according to the value of $\varphi$ and $l$, determine the coefficient of two one-dimensional filtering;

Step5: vertical to the ridge orientation, use one-dimensional band-pass filtering to filter the block, then the filtering image can be obtained;

Step6: along the ridge orientation use one-dimensional low-pass filtering to filtering image in step5, the final fingerprint enhancement image can be obtained.

\section{Conclusion}

Based on the traditional fingerprint image enhancement research, using the orientation selection and frequency selection characteristic of Gabor filtering, the fingerprint image orientation and the local ridge frequency is defined to the parameters of Gabor filtering function, the two-dimensional Gabor filtering is divided into a one-dimensional band-pass filtering and a one-dimensional low-pass filtering, the computing speed and execution efficiency are improved and has a good robustness. The effect fingerprint image enhancement largely depends on the quality of the input fingerprint, fingerprint aging, fuzzy has great influence on the fingerprint enhancement effect. In the future low quality fingerprint image enhancement research is the focus.

\section{Acknowledgement}

Our work is supported by project of science and technology development of Jilin provincial education department, China (No. 2012402).

Corresponding author: Hongchang Ke. Email: kehongchang1981@163.com.

\section{References}

[1] X. M. Cai, Y. J. Zhang, H. Liang. Modern Electronics Technique. vol. 24(2011) no. 12, p. 111. [2] Y. Chen,S. Dass, A. K. Jain. Proceedings of $5^{\text {th }}$ International Conference on Audio and Video based Biometric Person Authentication(New York, USA, 2005), p. 20.

[3] E. Lim, X. Jiang, W. Yau. Proeeedings of International Conference on Image Processing(New York, USA 2002), p. 469.

[4] J. Li, W. Y. Yau, H. Wang. Pattern Recognition. vol. 39(2006) no.1, p. $102 .$.

[5] L. Hong, Y. F. Wan, A. Jain. IEEE Transactions on Pattern Analysis and Machine Intelligence, vol. 20(1998)no. 8, p. 777.

[6] K. C. Chan, Y. S. Moon, P. S. Cheng. IEEE Transactions on Circuits and Systems for Video Technology, vol. 14(2004)no. 1, p. 95.

[7] J. Gu, J. Zhou, D. Zhang. Patten Recognition, vol. 37(2004)no. 3, p. 543. 